\title{
Flora fanerogâmica da Serra Negra, Minas Gerais, Brasil
}

\author{
Phanerogamic flora of Serra Negra, Minas Gerais, Brazil
}

Fátima Regina Gonçalves Salimena ${ }^{1,5}$, Carolina Nazareth Matozinhos ${ }^{2}$, Narjara Lopes de Abreu ${ }^{2}$, José Hugo Campos Ribeiro ${ }^{3}$, Filipe Soares de Souza ${ }^{3}$ \& Luiz Menini Neto ${ }^{1,4}$

\begin{abstract}
Resumo
O presente estudo teve como objetivo caracterizar a flora fanerogâmica da região da Serra Negra localizada no sul da Zona da Mata de Minas Gerais, entre os municípios de Lima Duarte, Rio Preto, Santa Bárbara do Monte Verde e Olaria. Embora considerada de importância biológica alta, esta região não possui nenhum registro anterior de dados florísticos, o que levou ao desenvolvimento deste levantamento, durante o período de 2003 a 2010. A vegetação é caracterizada por um mosaico de formações florestais e campestres onde se destacam os campos rupestres e florestas nebulares em altitudes que variam de 1300 a ca. $1700 \mathrm{~m}$. Um total de 1033 espécies foi encontrado, distribuídas em 469 gêneros e 121 famílias sendo as mais representativas Orchidaceae (115 spp.), Asteraceae 54 spp.), Melastomataceae (56 spp.), Myrtaceae (53 spp.), Fabaceae, Poaceae e Rubiaceae (48 spp. cada), Bromeliaceae (43 spp.), Solanaceae (38 spp.) e Piperaceae (33 spp). Novos registros e endemismos para a flora mineira foram encontrados e 58 espécies estão citadas na lista de espécies ameaçadas de Minas Gerais.

Palavras-chave: campo rupestre, Floresta Atlântica, floresta nebular, Serra da Mantiqueira, Zona da Mata Mineira.
\end{abstract}

\begin{abstract}
The present study aims to characterize the phanerogamic flora of the region of Serra Negra, located in the southern of Zona da Mata of Minas Gerais, between the municipalities of Lima Duarte, Rio Preto, Santa Bárbara do Monte Verde and Olaria. Although this region is considered of high biological importance, it has no previous record of floristic data, which led to the development of this survey, during the period 2003 to 2010. The vegetation is characterized by a mosaic of forests and grassland, especially cloud forests and "campos rupestres" (rocky grasslands), occurring at altitudes between 1300 to $1700 \mathrm{~m}$. A total of 1033 species was recorded, distributed in 469 genera and 121 families. The most representative families are Orchidaceae (115 spp.), Asteraceae 54 spp.), Melastomataceae (56 spp.), Myrtaceae (53 spp.), Fabaceae, Poaceae e Rubiaceae (48 spp. cada), Bromeliaceae (43 spp.), Solanaceae (38 spp.) and Piperaceae (33 spp). New records and endemic species to flora of Minas Gerais were found and 58 species are cited in the list of endangered species of Minas Gerais. Key words: Atlantic forest, "campo rupestre”, cloud forest, Mantiqueira Range, Zona da Mata of Minas Gerais.
\end{abstract}

\section{Introdução}

A Floresta Atlântica é a segunda maior floresta pluvial tropical do continente americano e uma das maiores do planeta, considerada Reserva da Biosfera pela UNESCO e hotspot de diversidade (Myers et al. 2000; Mittermeier et al. 2004; Silva $\&$ Casteleti 2005; Tabarelli et al. 2005). Juntamente com a Caatinga e o Cerrado, a Floresta Atlântica é responsável pela grande variedade de paisagens encontradas em Minas Gerais, favorecendo o elevado grau de riqueza florística do Estado (Mendonça \& Lins 2000; Drummond et al. 2005). Hoje restam apenas cerca de $8 \%$ da cobertura vegetacional original da Floresta Atlântica, resultado da ocupação desordenada do território, atividades agropecuárias, extrativismo vegetal, produção mineral e ação antrópica (Câmara 2005; Costa \& Herrmann 2006; Scolforo \& Carvalho 2006).

\footnotetext{
Este artigo possui material adicional em sua versão eletrônica.

${ }^{1}$ Universidade Federal de Juiz de Fora, Depto. Botânica, Campus Universitário, Martelos, 36036-900, Juiz de Fora, MG, Brasil

${ }^{2}$ Universidade Federal do Rio de Janeiro, Museu Nacional, Programa de Pós-graduação em Ciências Biológicas, $20940-040$ Rio de Janeiro, RJ, Brasil.

${ }^{3}$ Universidade Federal de Juiz de Fora, Programa de Pós-graduação em Ecologia, Campus Universitário, Martelos, 36036-900, Juiz de Fora, MG, Brasil.

${ }^{4}$ Centro de Ensino Superior de Juiz de Fora, R. Luz Interior, Santa Luzia, 36030-776, Juiz de Fora, MG, Brasil.

${ }^{5}$ Autora para correspondência: fatima.salimena@ufjf.edu.br
} 
As maiores extensões de florestas remanescentes em Minas Gerais são encontradas na Serra da Mantiqueira, maior e mais importante cadeia montanhosa do sudeste mineiro (Costa \& Herrmann 2006). Toda esta região serrana é considerada como área de importância biológica alta, devido à sua destacada diversidade, da qual há reduzido conhecimento científico (Drumonnd et al. 2005; Stehmann \& Sobral 2009). Neste sentido, os inventários biológicos são parte fundamental no entendimento dessa riqueza, constituindose, ainda nos dias de hoje, uma prioridade das pesquisas no Estado. De modo geral, ambientes montanos, como os encontrados na Serra da Mantiqueira, comumente apresentam mosaicos de vegetação campestre e florestal, abrigando alta diversidade e numerosos endemismos. Esta riqueza é freqüentemente atribuída a fatores climáticos e geológicos e às respectivas adaptações das espécies a condições extremas, porém o conhecimento aprofundado sobre a biodiversidade de tais ambientes é considerado escasso no território brasileiro (Martinelli 2007).

A região da Serra Negra integra o corredor sudeste do Complexo da Mantiqueira, o qual reúne remanescentes primários do domínio atlântico que apresentam alta conectividade, incluindo as florestas urbanas de Juiz de Fora e as áreas prioritárias de Bom Jardim de Minas, de Bocaina de Minas, de Monte Verde-APA Fernão Dias e do Parque Estadual do Ibitipoca (do qual dista cerca de $28 \mathrm{~km}$ ). Esta região é amplamente explorada tanto pela atividade agropecuária quanto pelo reflorestamento de Pinus L. e Eucalyptus L'Her., além da especulação imobiliária (Drummond et al. 2005). A despeito desta comprovada importância, é preocupante a quase inexistência de unidades de conservação nesta região, exceção feita a duas Reservas Particulares do Patrimônio Natural (RPPNs), sendo uma na face sul da Serra, no Vilarejo do Funil, no município de Rio Preto (RPPN São Lourenço do Funil) e a outra na face norte, no município de Lima Duarte (RPPN Serra Negra).

O reconhecimento da importância biológica da Serra Negra como parte do Complexo da Mantiqueira no sul da Zona da Mata de Minas Gerais, corroborada por estudos decorrentes de um amplo levantamento florístico, realizado até 2010 (Abreu et al. 2007, 2011; Batista et al. 2008; Menini Neto et al. 2009; Abreu \& Menini Neto 2010; Assis \& Mello-Silva 2010; Feliciano \& Salimena 2011; Matozinhos \& Konno 2011; Valente et al.
2011; Dutra et al. (2012); Blaser et al. (2012); Mezzonato-Pires et al. (2013)), além de um sítio virtual de divulgação da riqueza florística desta área (< http://www.ufjf.br/floraserranegra/>), levou ao desenvolvimento deste inventário a partir de 2003.

O presente trabalho tem como objetivos apresentar inventário florístico das fanerógamas ocorrentes na Serra Negra, caracterizar a vegetação ocorrente e discutir a conservação da área e a relação desta com outras áreas de vegetação predominantemente campestre no estado de Minas Gerais.

\section{Material e Métodos}

Área de estudo

A Serra Negra localiza-se no sul da Zona da Mata (21 ${ }^{\circ} 58^{\prime} 11^{\prime \prime S}, 43^{\circ} 53$ '21'W), integrando o Complexo da Mantiqueira, sob o Domínio Atlântico. Está limitada pelos municípios de Lima Duarte (ao norte), Rio Preto (ao sul), Santa Bárbara do Monte Verde (a leste) e Olaria (a oeste), posicionando-se a leste da Área de Proteção Ambiental da Serra da Mantiqueira, próximo à divisa com o estado do Rio de Janeiro (Fig. 1).

Apresenta altitudes entre 800 e ca. $1700 \mathrm{~m}$ e o relevo é marcadamente montanhoso (Fig. 2a) com escarpas íngremes e vales encaixados, apresentando cristas e picos elevados que ultrapassam $1400 \mathrm{~m}$ de altitude, destacados do domínio geomorfológico predominante de morros e colinas rebaixadas que caracteriza a porção mais dissecada do sopé da vertente sul da Mantiqueira (Heilbron et al. 2000). A geologia da região é composta predominantemente pelo Grupo Andrelândia, com solos do tipo Latossolo Amarelo Distrófico Típico e Latossolo Amarelo Distrófico Argissólico,

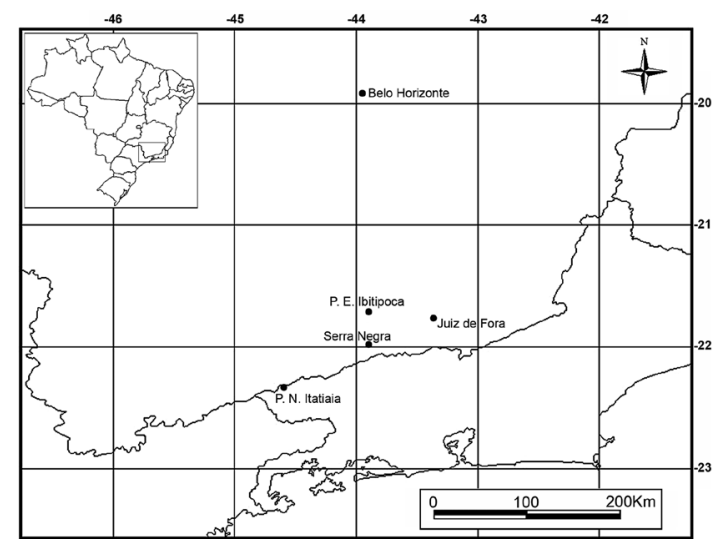

Figura 1 - Localização da Serra Negra, Minas Gerais, Brasil. Figure 1 - Location of Serra Negra, Minas Gerais, Brazil. 
além da presença de material arenoso (quartzito), tendo como característica baixa fertilidade natural (Olszevski et al. 2008). A unidade geológica deste grupo inclui rochas metassedimentares de idade pré-cambriana, cuja geomorfologia cárstica vem sendo estudada nos últimos anos com a descrição de quartzito grosseiro, quartzito fino e gnaisse bandado na região (Avelar et al. 2006; Uagoda et al. 2006).

O clima da região é do tipo Cwb (Köppen) com média de precipitação anual de $1.886 \mathrm{~mm}$ (EMATER 2003).

\section{Metodologia}

Foram realizadas expedições de coleta entre os anos de 2003 e 2010, que buscaram cobrir a maior área possível da Serra Negra, por trilhas preexistentes e outras abertas na área de estudo. Os exemplares férteis foram fotografados em campo, coletados segundo a metodologia proposta por Mori et al. (1990) e os dados relevantes para cada espécime, como coloração, hábito e ambiente de ocorrência, foram anotados em campo. Os espécimes foram herborizados segundo a metodologia usual e depositados no herbário CESJ, da Universidade Federal de Juiz de Fora (acrônimo de acordo com Thiers (2011)) e duplicatas enviadas a diversos herbários pertencentes ao programa de permuta do herbário CESJ.

As identificações foram feitas por comparação com exemplares do acervo do herbário CESJ e consulta à bibliografia especializada. Os nomes de espécies e autores foram conferidos através de consulta ao sítio The Plant List $(<\mathrm{http}$ ://www. theplantlist.org/>) e as famílias estão de acordo com APG III (2009). Não foram consideradas categorias subespecíficas. Os dados das espécies ameaçadas de extinção no estado de Minas Gerais foram obtidos no sítio da Fundação Biodiversitas (<http://www. biodiversitas.org.br/listas-mg $>$ ).

\section{Resultados e Discussão}

\section{Caracterização da vegetação}

A vegetação é formada por um mosaico de fragmentos com fisionomias florestais, arbustivas, campestres e áreas antrópicas (estas últimas representadas principalmente por estradas, pastagens e plantações). Historicamente a área foi utilizada para extração de madeiras e criação de gado leiteiro (atividade que se encontra em declínio), explicando, assim, a ocorrência das pastagens com gramíneas (Uagoda et al. 2006).
Os fragmentos florestais foram caracterizados por Valente et al. (2011) como: Floresta Ombrófila Densa Aluvial, Floresta Ombrófila Densa Montana e Floresta Ombrófila Densa Alto-Montana (Floresta Nebular). Além destas tipologias, podem ser encontrados também fragmentos de Floresta Estacional Semidecidual em algumas áreas em torno de 900-1000 m de altitude.

A Floresta Ombrófila Densa Aluvial é representada pelo fragmento localmente conhecido como Mata de Cambuí. É uma floresta a cerca de $900 \mathrm{~m}$ de altitude, sazonalmente inundável pela elevação do lençol freático do Ribeirão do Funil. Apresenta dossel com monodominância de Myrciaria tenella (DC.) O.Berg (Myrtaceae) ("cambuí) (Fig. 2b). Observam-se extensos trechos do interior da mata de cambuí em que o sub-bosque é inexistente, havendo somente uma vegetação herbácea rasteira composta principalmente por Lithachne horizontalis Chase (Poaceae) e pelas samambaias Asplenium martianum C.Chr. (Aspleniaceae) e Thelypteris spp. (Thelypteridaceae). Há também a presença de algumas epífitas, como Hatiora salicornioides (Haw.) Britton \& Rose e Rhipsalis baccifera (J.S.Muell.) Stearn (Cactaceae), Sophronitis cernua Lindl. (Orchidaceae), Microgramma tecta (Kaulf.) Alston e trepadeiras como Lygodium volubile Sw. (Lygodiaceae) e Mendoncia velloziana Mart. (Acanthaceae).

Ao longo de toda a Serra, até os $1500 \mathrm{~m}$ de altitude ocorrem fragmentos de Floresta Ombrófila Densa Montana que apresentam a predominância de árvores de médio a grande porte, formando um dossel contínuo de até $20 \mathrm{~m}$ de altura, com algumas espécies emergentes (Fig. 2c). Destacase um fragmento de aproximadamente 0,9 ha conhecido na região como Cânion do Funil, devido à expressiva riqueza de espécies vasculares nãoarbóreas, sobretudo epífitas (Menini Neto et al. 2009). A partir de $1500 \mathrm{~m}$ a Floresta Ombrófila Densa Alto-Montana (Fig. 2d), apresenta dossel mais baixo em relação à Floresta Ombrófila Densa Montana e alta umidade. Destaca-se a ocorrência de espécies arbóreas de Myrtaceae, Melastomataceae, Lauraceae, Rubiaceae, Solanaceae e Nyctaginaceae e abundância de epífitos, principalmente Orchidaceae, Bromeliaceae, Cactaceae e Piperaceae (como representantes de Angiospermas) e Polypodiaceae (entre as samambaias) (Fig. 2e). A Floresta Estacional Semidecidual ocupa as encostas da Serra, entre aproximadamente 800 e 1100 m 

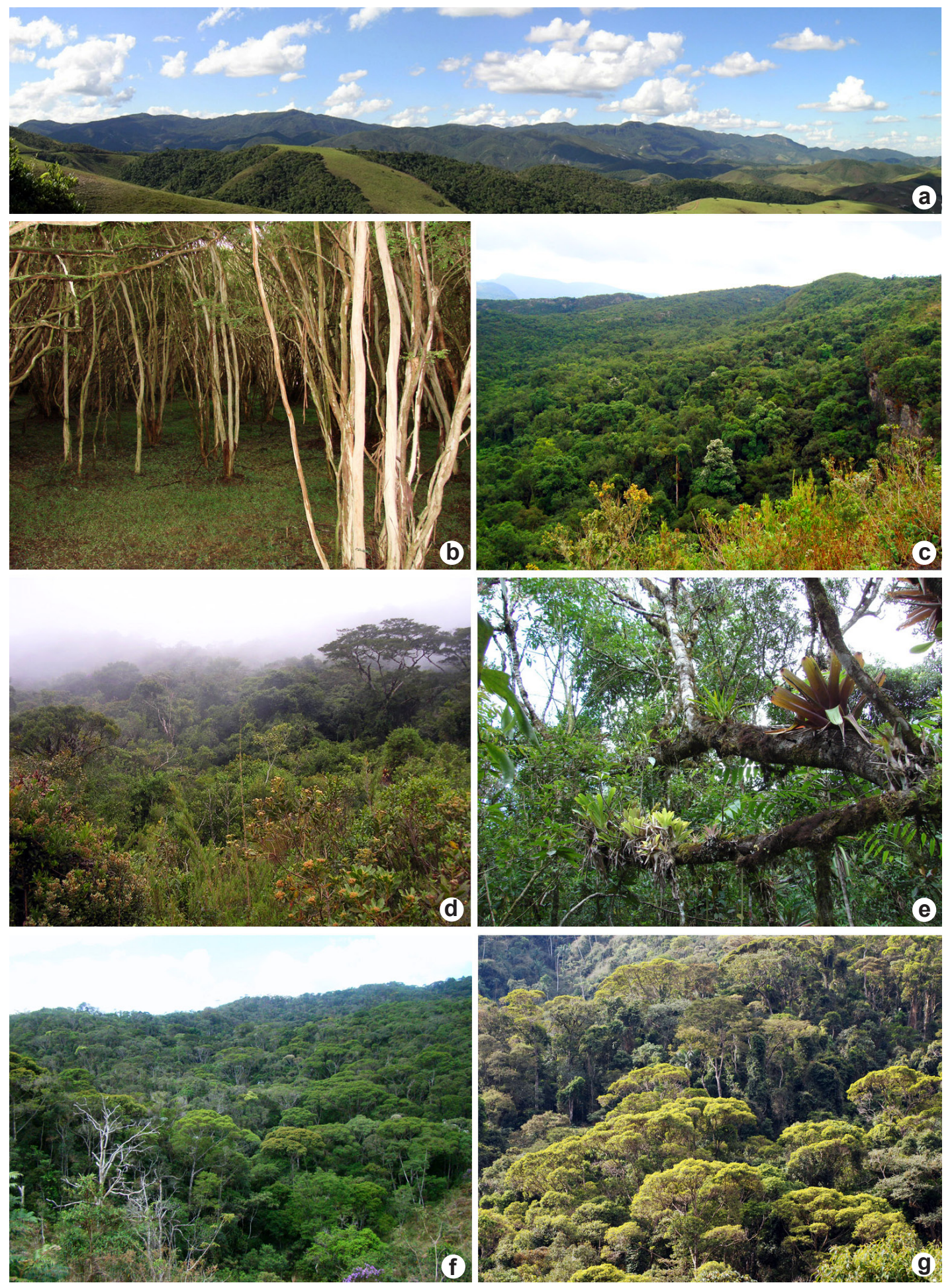

Figura 2 - a-g. tipo de vegetação encontrada na Serra Negra - a. vista panorâmica da face sul da Serra Negra; b. Floresta Ombrófila Densa Aluvial (“Cambuí”); c. Floresta Ombrófila Densa Montana; d-e. Floresta Ombrófila Densa Altomontana (Floresta Nebular), d. vista geral, e. vista do interior, com detalhe de ocorrência de epífitas. f-g. Floresta Estacional Semidecidual. Figure 2 - a-g. vegetation types occurring in Serra Negra - a. panoramic view of southern side of Serra Negra; b. Alluvial Dense Rainforest (“Cambuí"); c. Montane Dense Rainforest; d-e. High Montande Dense Rainforest (Cloud Forest), d. general view, e. interior view, with detail on epiphytic plants. f-g. Seasonal Semidecidual Forest. 
de altitude (Fig. 2f-g). As espécies arbóreas mais representativas pertencem às famílias Fabaceae, Melastomataceae e Myrtaceae.

A vegetação com físionomia arbustiva está presente ao longo de toda a área, sobretudo nas encostas da serra, sobre afloramentos de rochas quartzíticas e mais comumente sobre areia branca resultante da erosão destas rochas. Predominam arbustos de até 1,8 m altura, ocorrendo em diferentes graus de adensamento e em grande parte da área são encontradas amplas populações entremeadas de "candeias" (Eremanthus erythropappus (DC.) MacLeish e E. glomerulatus Less., Asteraceae) (Fig. 3a-b). Ocorrem ainda algumas epífitas e um estrato composto por muitas herbáceas, musgos e liquens, que no entanto não é contínuo. São comuns espécies de Aquifoliaceae, Celastraceae, Fabaceae, Malpighiaceae, Melastomataceae e Myrtaceae, dentre outras. Esta fisionomia é equivalente ao escrube segundo Eiten (1979) ou ao arbustal latifoliado segundo classificação proposta por Oliveira-Filho (2009).

A outra fisionomia não florestal é representada pelos campos rupestres, que ocorrem de forma esparsa por toda a área em afloramentos quartzíticos, associados à areia branca (Fig. 3c-f). São comuns principalmente espécies de Poaceae, Cyperaceae e Eriocaulaceae. Ocorrem em maiores extensões nas cumeeiras da Serra, apresentando frequentemente estrato herbáceo contínuo, interrompido em alguns pontos por afloramentos de rocha nua, composto principalmente por gramíneas e presença de alguns arbustos e raras arvoretas, além de extensa população de Chusquea riosaltensis L.G.Clark (Poaceae) (Fig. 3g-h).

\section{Florística}

Foram coletados cerca de 2700 exemplares de fanerógamas neste estudo, sendo registradas 1033 espécies, distribuídas em 469 gêneros e 121 famílias, sendo 119 de Angiospermas e apenas duas de Gimnospermas (Apêndice), representadas por uma espécie cada e encontradas de forma esporádica na região estudada: Araucariaceae (Araucaria angustifolia (Bertol.) Kuntze) e Podocarpaceae (Podocarpus lambertii Klotzsch ex Endl.).

A família de maior riqueza específica na Serra Negra é Orchidaceae (115 spp.) seguida por Asteraceae (54 spp.), Melastomataceae (56 spp.), Myrtaceae (53 spp.), Fabaceae, Poaceae e Rubiaceae (48 spp. cada), Bromeliaceae (43 spp.), Solanaceae (38 spp.) e Piperaceae (33 spp). Assim, apenas 10 famílias somam um total de 536 espécies, equivalente a cerca de $52 \%$ da flora fanerogâmica da área de estudo.

Estas famílias, de modo geral, são também as mais representativas em estudos realizados em áreas com predominância de fisionomia campestre no estado de Minas Gerais como: Serra do Cipó (Giulietti et al. 1987), Grão Mogol (Pirani et al. 2003), Parque Estadual da Serra do Brigadeiro (Leoni \& Tinte 2004), Serra de São José (Alves \& Kolbek 2009) e Parque Estadual do Ibitipoca (Forzza et al. dados não publicados). Cinco aparecem entre as dez mais ricas em todos estes estudos: Orchidaceae (monocotiledônea), Asteraceae, Fabaceae, Melastomataceae e Rubiaceae (eudicotiledôneas). Com exceção de Melastomataceae, estas são as famílias de angiospermas de maior riqueza específica em termos globais e também as mais representativas na flora do Brasil, ficando Melastomataceae, neste caso, na sexta posição (Forzza et al. 2010).

Os gêneros mais ricos foram Solanum L. (Solanaceae) com 22 espécies, seguido por Myrcia DC. (Myrtaceae) com 21 spp., Piper L. (Piperaceae) com 19 spp., Miconia Ruiz \& Pav. (Melastomataceae) com 16 spp., Eugenia L. (Myrtaceae) com 15 spp., Vriesea Lindl. (Bromeliaceae) e Peperomia Ruiz \& Pav. (Piperaceae) com 14 spp. cada, Leandra Raddi (Melastomataceae) e Epidendrum L. (Orchidaceae) com 13 spp. cada, Psychotria L. (Rubiaceae) com 12 spp., Passiflora L. (Passifloraceae) com 11 spp. e Oncidium s.l. Sw. (Orchidaceae) com 10 spp.

A partir de coletas feitas neste estudo espécies novas foram descritas, como Habenaria pseudoglaucophylla J.A.N.Batista, R.C.Mota \& N.L.Abreu (Orchidaceae) (Batista et al. 2008), Ocotea colophanthera L.C.S.Assis \& Mello-Silva e O. rupestris L.C.S.Assis \& MelloSilva (Lauraceae) (Assis \& Mello-Silva 2010) e Macroditassa mantiqueirae Matozinhos \& T.U.P.Konno (Apocynaceae) (Matozinhos \& Konno 2011), e exemplares pertencentes aos gêneros Anthurium Schott (Araceae), Passiflora L. (Passifloraceae), Plinia L. (Myrtaceae) e Solanum L. (Solanaceae) encontram-se em estudo, podendo também representar novas espécies para a ciência.

O conhecimento da distribuição geográfica de algumas espécies foi ampliado, com novos registros de ocorrência feitos para o estado de Minas Gerais, como: Maxillaria bradei Schltr. ex Hoehne e Pabstia jugosa (Lindl.) Garay (Orchidaceae) 
(Abreu et al. 2007), Eugenia moonioides O.Berg (Myrtaceae) e Heterocondylus jaraguensis (B.L.Rob.) R.M.King \& H.Rob. (Asteraceae). Em menor escala, houve a ampliação do conhecimento de distribuição de espécies antes restritas ao Parque Estadual do Ibitipoca, do qual a Serra Negra dista cerca de $28 \mathrm{Km}$ : Chusquea riosaltensis (Poaceae), Hindsia ibitipocensis Di Maio (Rubiaceae) e Vriesea cacuminis L.B.Sm. (Bromeliaceae).

Até o momento, são consideradas endêmicas da região da Serra Negra Aechmea bruggeri Leme (Bromeliaceae), Macroditassa mantiqueirae, Ocotea rupestris, Vanhouttea brueggeri Chautems e $V$. hilariana Chautems (Gesneriaceae).

Dentre as fanerógamas registradas na Serra Negra, 58 são citadas na lista de espécies ameaçadas de Minas Gerais (<http://www. biodiversitas.org.br/listas-mg $>$ ) nas categorias, "Em Perigo" (EN) (17 spp.), "Vulnerável" (VU) (14 spp.), "Deficiente de Dados" (DD) (13 spp.), "Criticamente em Perigo" (CR) (oito spp.) e "Quase Ameaçada" (NT) (seis spp.), distribuídas em 25 famílias, das quais se destacam Orchidaceae (10 spp.), Bromeliaceae (sete spp.) e Gesneriaceae (quatro spp.). Estes dados reforçam a importância do estabelecimento de alguma forma de proteção na região, uma vez que as únicas Unidades de Conservação são duas RPPN's responsáveis por resguardar apenas uma pequena parcela da biodiversidade existente na Serra Negra. Outras 33 espécies encontradas na Serra Negra são consideradas como "Não ameaçadas" (LC), pois seu status foi revisado em relação à lista vermelha apresentada por Mendonça \& Lins (2000) (ver Apêndice na versão on line deste trabalho).

Embora os campos rupestres sejam ambientes mais freqüentemente encontrados na Cadeia do Espinhaço em Minas Gerais e Bahia (Giulietti et al. 1987, 2000), algumas disjunções podem ser observadas em Goiás e nas regiões sudoeste (Serra da Canastra), sul e sudeste de Minas Gerais (incluindo, por exemplo, a Serra de Carrancas, Serra de São José, Serra do Ibitipoca, Serra de Santa Maria do Baependi) (Romero 2002; Alves \& Kolbek 2009; Ferreira \& Forzza 2009).

A região da Serra Negra aparentemente atua como uma área de tensão ecológica ou ecótono, pois é possível reconhecer em nível genérico ou específico elementos florísticos exclusivos ou predominantemente distribuídos tanto nos campos rupestres ao norte, quanto nos campos de altitude ao sudoeste e leste da área.
Alguns destes elementos florísticos encontrados, por exemplo, na Serra do Ibitipoca, Serra de São José ou em campos rupestres da Cadeia do Espinhaço (Giulietti et al. 1987, Pirani et al. 2003, Alves \& Kolbek 2009) são os que seguem: Ditassa mucronata Mart. (Apocynaceae) Eremanthus erythropappus, E. glomerulatus (Asteraceae), Vriesea cacuminis (Bromeliaceae), Arthrocereus melanurus (K.Schum.) Diers, P.J.Braun \& Esteves (Cactaceae), Paepalanthus manicatus Poulsen ex Malme (Eriocaulaceae), Cambessedesia hilariana DC., Microlicia fulva (Spreng.) Cham. (Melastomataceae), Hoffmannseggella crispata (Thunb.) H.G.Jones, Bulbophyllum exaltatum Lindl. (Orchidaceae), Hindsia ibitipocensis (Rubiaceae), Barbacenia flava Mart. ex Schult. f. e Barbacenia tomentosa Mart. (Velloziaceae).

Quanto aos táxons compartilhados com os ambientes campestres dos campos de altitude ou áreas florestais associadas na região Sudeste do Brasil, como Serra do Brigadeiro, Macaé de Cima e Itatiaia (Brade 1956; Lima \& Guedes-Bruni 1996, Leoni \& Tinte 2004), podem ser citados, dentre outros: Ditassa conceptionis Fontella, Mandevilla pendula (Ule) Woodson (Apocynaceae), Scybalium glaziovii Eichler (Balanophoraceae), Siphocampylus longepedunculatus Pohl (Campanulaceae), Ormosia altomontana J.E.Meireles \& H.C.Lima (Fabaceae), Behuria parvifolia Cogn., Huberia nettoana Brade, Meriania claussenii (Naudin) Triana (Melastomataceae), Xyris fusca L.A.Nilson (Xyridaceae).

Ainda, algumas espécies registradas na Serra Negra são encontradas em ambientes montanos tanto nos campos rupestres quanto nos campos de altitude (Safford 2007, Garcia \& Pirani 2005), destacando-se Clethra scabra Pers. (Clethraceae), Lamanonia ternata Vell. (Cunoniaceae), Fuchsia regia (Vell.) Munz (Onagraceae), Podocarpus lambertii Klotzsch ex Endl. (Podocarpaceae) e Drimys brasiliensis Miers (Winteraceae), sendo que as angiospermas são consideradas elementos de origem andina.

Deste modo, a vegetação da Serra Negra recebe influências das floras de outras localidades contíguas da região Sudeste do Brasil e, embora não exista uma análise de similaridade entre estas áreas empregando a flora completa, alguns estudos com grupos específicos realizados na Serra Negra reforçam esta conclusão.

As pteridófitas apresentaram alta similaridade, entre 0,59 e 0,64, com a Serra do Caraça, Parque 

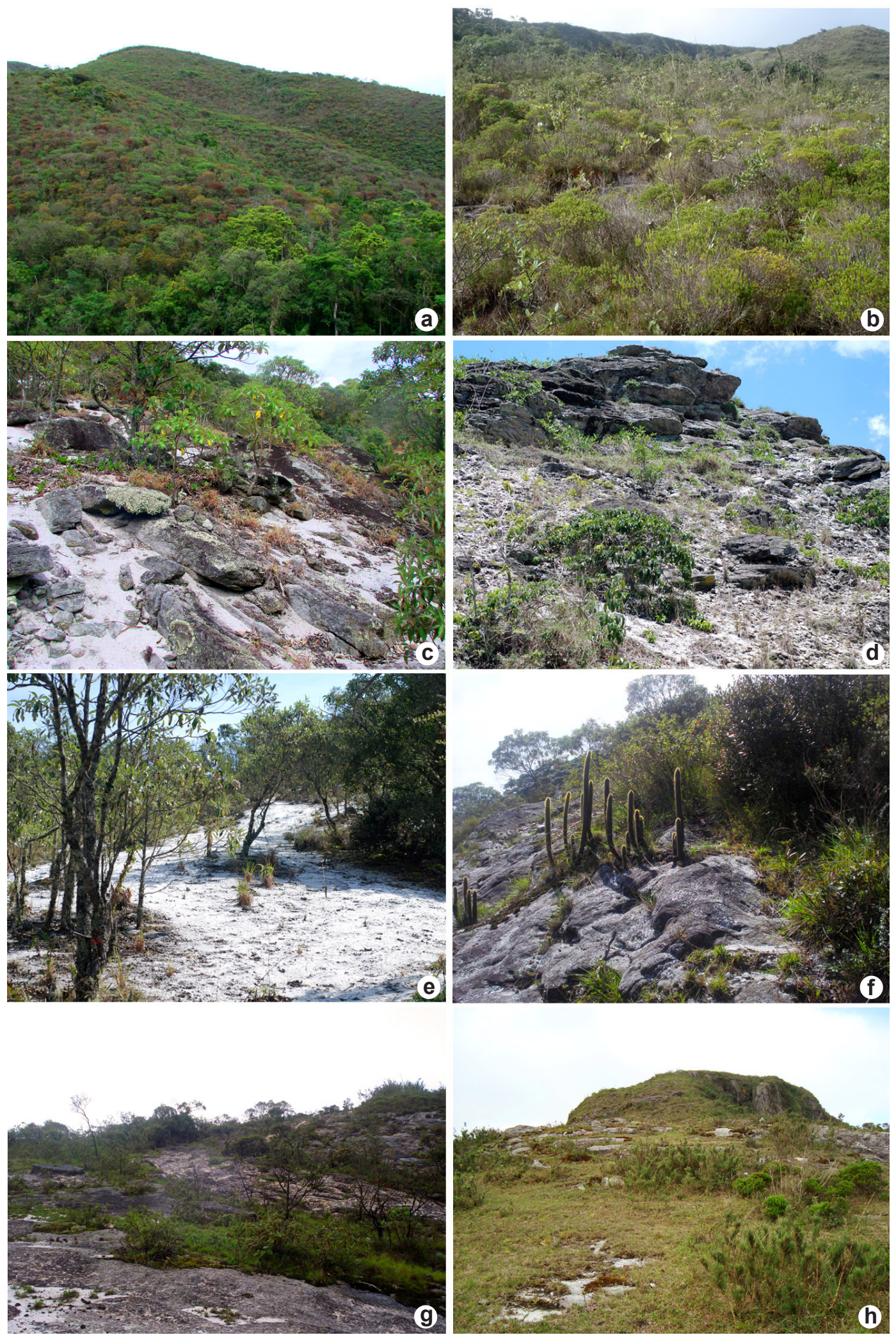

Figura 3 - a-b. Vegetação arbustiva (escrube ou arbustal latifoliado); c-h. Campo rupestre.

Figure 3 - a-b. Shrubby vegetation (scrub or broadleaved scrub); c-h. "Campo rupestre" (rocky grassland). 
Estadual de Ibitipoca e Parque Estadual do Itacolomi (nesta ordem) conforme apresentado por Souza et al. (2012). De modo semelhante Abreu et al. (2011) em estudo das Orchidaceae da Serra Negra obtiveram maior similaridade com o Parque Estadual de Ibitipoca $(0,64)$ e Serra do Caraça $(0,54)$. Em ambos os casos, a similaridade é maior com áreas predominantemente campestres que apresentam florestas entremeadas em maior ou menor extensão.

No entanto, para a família Apocynaceae estudada por Matozinhos (2010) descreve maior similaridade foi com áreas de fisionomia exclusivamente florestal, Reserva Biológica da Represa do Grama (composta por Floresta Estacional Semidecidual, município de Descoberto, Minas Gerais) e Parque Estadual das Fontes do Ipiranga (composto por Floresta Ombrófila Densa, no municípío de São Paulo). Para Solanaceae houve maior similaridade (embora não tão alta, com índice de ca. 0,4) com um grupo formado pelo Parque Estadual das Fontes de Ipiranga e Parque Nacional do Itatiaia (sendo este último composto predominantemente por campo de altitude), sugerindo influência variada na composição da família na Serra Negra (Feliciano 2008).

Nos estudos supracitados incluindo as famílias Apocynaceae, Orchidaceae e Solanaceae análises específicas sugerem que características ambientais como o tipo vegetacional, altitude e clima exercem influência sobre a similaridade florística entre as áreas. No caso de Orchidaceae, a análise de autocorrelação espacial demonstrou que distância geográfica não influenciou a similaridade florística entre as áreas analisadas. Os resultados obtidos para as pteridófitas parecem apontar para a mesma conclusão, embora, neste caso, nenhuma análise tenha sido feita neste sentido.

Por conseguinte, as relações florísticas entre a área estudada e as demais áreas da Região Sudeste do Brasil ainda não estão completamente esclarecidas e poderão ser mais bem entendidas com a realização de um estudo fitogeográfico mais aprofundado e abrangente e incluindo análises que contemplem variação altitudinal, latitudinal, pluviométrica e de interiorização das áreas, potencialmente importantes na interpretação destas relações.

\section{Agradecimentos}

À Fundação de Amparo à Pesquisa do Estado de Minas Gerais - FAPEMIG (Processos CRA 1891/06 e CRA 1810-5.02/07) e ao Conselho
Nacional de Pesquisa - CNPq (Processo 551462/2008-6) o apoio financeiro concedido para o desenvolvimento deste projeto. Aos especialistas que auxiliaram nas identificações das espécies. Aos proprietários das áreas visitadas na região da Serra Negra a autorização de coleta e o apoio nos trabalhos de campo. À Pró-Reitoria de Pesquisas da Universidade Federal de Juiz de Fora o transporte da equipe até a Serra Negra.

\section{Referências}

Abreu, N.L. \& Menini Neto, L. 2010. As subfamílias Vanilloideae e Orchidoideae (Orchidaceae) em um fragmento da Serra da Mantiqueira, Minas Gerais, Brasil. Boletim de Botânica da Universidade de São Paulo 28: 15-33.

Abreu, N.L.; Menini Neto, L. \& Konno, T.U.P. 2011. Orchidaceae das Serras Negra e do Funil, Rio Preto, Minas Gerais, e similaridade florística entre formações campestres e florestais do Brasil. Acta Botanica Brasilica 25: 58-70.

Abreu, N.L.; Santiago, A.L. \& Menini Neto, L. 2007. Novos registros de Orchidaceae para a flora do estado de Minas Gerais, Brasil. Orchidstudium International Journal of Orchid Study 2: 37-43.

Alves, R.J.V. \& Kolbek, J. 2009. Summit vascular flora of Serra de São José, Minas Gerais, Brazil. Check List 5: 35-73.

APG III. 2009. An update of the Angiosperm Phylogeny Group classification for the orders and families of flowering plants: APG III. Botanical Journal of the Linnean Society 161: 105-121.

Assis, L.C.S. \& Mello-Silva, R. 2010. Three new species of Ocotea (Lauraceae) from the campos rupestres of Brazil. Brittonia 62: 86-94.

Avelar, A.S.; Coelho Netto, A.L.; Uagoda, R.E.S. \& Ferro, C.S.R. 2006. Geologia, dissolução de rochas quartzíticas e formas cársticas na bacia do Ribeirão Santana (MG/RJ). In: Anais do VI Simpósio Nacional de Geomorfologia/Regional Conference on Geomorphology, Goiânia. Disponível em <http:// www.labogef.iesa.ufg.br/links/sinageo/articles/230. pdf $>$. Acesso em 20 Set 2011.

Batista, J.A.N.; Mota, R.C.; Abreu, N.L. \& Menini Neto, L. 2008. Habenaria pseudoglaucophylla (Orchidaceae), a new species from Minas Gerais, Brazil. Novon 18: 409-414.

Blaser, J.G.; Salimena, F.R.G. \& Chautems, A. 2012. Gesneriaceae na Serra Negra, Minas Gerais, Brasil. Rodriguésia 63: 705-714.

Brade, A.C. 1956. A flora do Parque Nacional do Itatiaia. Boletim do Parque Nacional do Itatiaia 5: 7-85.

Câmara, I.G. 2005. Breve história da conservação da Mata Atlântica. In: Galindo-Leal, C. \& Câmara, I.G. (eds.). Mata Atlântica: biodiversidade, ameaças e perspectivas. Conservação Internacional, Belo Horizonte. Pp. 31-42. 
Costa, C. \& Herrmann, G. 2006. Plano de ação do Corredor Ecológico da Mantiqueira. Valor Natural, Belo Horizonte. 64p.

Drummond, G.M.; Martins, C.S.; Machado, A.B.M.; Sebaio, F.A. \& Antonini, Y. 2005. Biodiversidade em Minas Gerais, um atlas para sua conservação. $2^{\mathrm{a}}$ ed. Fundação Biodiversitas, Belo Horizonte. 222p.

Eiten, G. 1979. Formas fisionômicas do Cerrado. Revista Brasileira de Botânica 2: 139-148.

Empresa de Assistência Técnica e Extensão Rural do Estado de Minas Gerais - EMATER. 2003. Projeto APA da Serra do Funil. EMATER, Rio Preto. 63p.

Feliciano, E.A. 2008. Solanaceae A. Juss. da Serra Negra, Rio Preto, Minas Gerais: tratamento taxonômica e similaridade florística. Dissertação de Mestrado. Universidade Federal de Juiz de Fora, Juiz de Fora. 135p.

Feliciano, E.A. \& Salimena, F.R.G. 2011. Solanaceae na Serra Negra, Rio Preto, Minas Gerais. Rodriguésia 62: 1-22.

Ferreira, F.M. \& Forzza, R.C. 2009. Florística e caracterização da vegetação da Toca dos Urubus, Baependi, Minas Gerais, Brasil. Biota Neotropica 9: 131-148.

Forzza, R.C.; Baumgratz, J.F.A.; Costa. A.; Hopkins, M.; Leitman, P.M.; Lohmann, L.G.; Martinelli, G.; Morim, M.P.; Coelho, M.A.N.; Peixoto, A.L.; Pirani, J.R.; Queiroz, L.P.; Stehmann, J.R.; Walter, B.M.T. \& Zappi, D. 2010. As angiospermas do Brasil. In: Forzza, R.C.; Baumgratz, J.F.A.; Bicudo, C.E.M.; Carvalho Jr., A.A.; Costa, A.; Costa, D.P.; Hopkins, M.; Leitman, P.M.; Lohmann, L.G.; Maia, L.C.; Martinelli, G.; Menezes, M.; Morim, M.P.; Coelho, M.A.N.; Peixoto, A.L.; Pirani, J.R.; Prado, J.; Queiroz, L.P.; Souza, V.C.; Stehmann, J.R.; Sylvestre, L.S.; Walter B.M.T. \& Zappi D. (eds.). Catálogo de plantas e fungos do Brasil. Jardim Botânico do Rio de Janeiro, Rio de Janeiro. Pp. 19-42.

Garcia, R.J.F. \& Pirani, J.R. 2005. Análise florística, ecológica e fitogeográficado Núcleo Curucutu, Parque Estadual da Serra do Mar (São Paulo, SP), com ênfase nos campos junto à crista da Serra do Mar. Hoehnea 32: 1-48.

Giulietti, A.M.; Menezes, N.L.; Pirani, J.R.; Meguro, M. \& Wanderley, M.G.L. 1987. Flora da Serra do Cipó, Minas Gerais: Caracterização e lista das espécies. Boletim de Botânica da Universidade de São Paulo 9: 1-151.

Giulietti, A.M.; Harley, R.M.; Queiroz, L.P.; Wanderley, M.G.L. \& Pirani, J.R. 2000. Caracterização e endemismos nos campos rupestres da Cadeia do Espinhaço. In: Cavalcanti, T.B. \& Walter, B.M.T. (eds.). Tópicos atuais em botânica. EMBRAPA Recursos Genéticos, Brasília. Pp. 311-318.

Heilbron, M.; Tupinambá, M.; Eirado, L.G.; Rribeiro, A.; Paciullo, F.V.P.; Trouw, R.A.; Valeriano, C.M.; Junho, M.C.B.; Roig, H.L.; Nogueira, J.R.; Medeiros, R.M.; Rocha, D.; Polonia, J.; Silva,
R.R. \& Toledo, C. 2000. Geologia das folhas Santa Rita do Jacutinga e Rio Preto. Relatório técnico. COMIG-UFMG-UFRJ-UERJ.

Leoni, L.S. \& Tinte, V.A. 2004. Flora do Parque Estadual da Serra do Brigadeiro, estado de Minas Gerais, Brasil - Caracterização da vegetação e lista preliminar das espécies. Gráfica São José, Carangola. 91p.

Lima, M.P.M. \& Guedes-Bruni, R.R. (orgs.). 1996. Reserva Ecológica de Macaé de Cima: Nova Friburgo-RJ - Aspectos florísticos das espécies vasculares. Jardim Botânico do Rio de Janeiro, Rio de Janeiro. 465p.

Martinelli, G. 2007. Mountain biodiversity in Brazil. Revista Brasileira de Botânica 30: 587-597.

Matozinhos, C.N. 2010. Apocynaceae da Serra Negra, Minas Gerais, Brasil: diversidade taxonômica e distribuição espacial no sudeste do Brasil. Dissertação de Mestrado. Universidade Federal do Rio de Janeiro/Museu Nacional, Rio de Janeiro. $135 \mathrm{p}$.

Matozinhos, C.N. \& Konno, T.U.P. 2011. A new species of Macroditassa (Apocynaceae-Asclepiadoideae) from Minas Gerais, Brazil. Systematic Botany 36: 137-140.

Mendonça, M.P. \& Lins, L.V. 2000. Lista vermelha das espécies ameaçadas de extinção da flora de Minas Gerais. Fundação Biodiversitas, Belo Horizonte. 157p.

Menini Neto, L.; Matozinhos, C.N.; Abreu, N.L.; Valente, A.S.M.; Antunes, K.; Souza, F.S.; Viana, P.L. \& Salimena, F.R.G. 2009. Flora vascular não-arbórea de uma floresta de grota na Serra da Mantiqueira, Zona da Mata de Minas Gerais, Brasil. Biota Neotropica 9: 1-13.

Mezzonato-Pires, A.C.; Salimena, F.R.G. \& Bernacci, L.C. 2013. Passifloraceae na Serra Negra, Minas Gerais, Brasil. Rodriguésia 64: 123-136.

Mittermeier, R.A.; Gil, P.R.; Hoffmann, M.; Pilgrim, J; Brooks, T.; Mittermeier, C.G.; Lamoreux, J. \& Fonseca, G.A. B. 2004. Hotspots revisited: Earth's biologically richest and most endangered terrestrial ecoregions. CEMEX, Mexico City. 392p.

Mori, S.; Mattos-Silva, L.A.; Lisboa, G. \& Coradin, L. 1990. Manual de manejo do herbário fanerogâmico. 2ed. Centro de Pesquisas do Cacau, Ilhéus. 103p.

Myers, N.; Mittermeier, R.A.; Mittermeier, C.G.; Fonseca, G.A.B. \& Kent, J. 2000. Biodiversity hotspots for conservation priorities. Nature 403: 853-858.

Oliveira-Filho, A.T. 2009. Classificação das fitofisionomias da América do Sul cisandina tropical e subtropical: proposta de um novo sistema - prático e flexível - ou uma injeção a mais de caos? Rodriguésia 60: 237-258.

Olszevski, N.; Costa, L.M.; Fernandes Filho, E.I. \& Costa, O.D.V. 2008. Paisagem e uso da terra em diferentes unidades geológicas em área sob influência do Rio Preto (MG-RJ). Revista de Biologia e Ciências da Terra 8: 45-59. 
Pirani, J.R.; Mello-Silva, R. \& Giulietti, A.M. 2003. Flora de Grão Mogol, Minas Gerais, Brasil. Boletim de Botânica da Universidade de São Paulo 21: 1-24.

Romero, R. 2002. Diversidade da flora dos campos rupestres de Goiás, sudoeste e sul de Minas Gerais. In: Araújo, E.L.; Moura, A.N.; Sampaio, E.V.S.B; Gestinari, L.M.S. \& Carneiro, J.M.T. (eds.). Biodiversidade, conservação e uso sustentável da flora do Brasil. Universidade Federal Rural de Pernambuco, Recife. Pp. 81-86.

Safford, H.D. 2007. Brazilian Páramos IV. Phytogeography of the campos de altitude. Journal of Biogeography 34: 1701-1722.

Scolforo, J.R.S. \& Carvalho, L.M.T. 2006. Mapeamento e inventário da flora nativa e dos reflorestamentos de Minas Gerais. Ed. UFLA, Lavras. 288p.

Silva, J.M.C. \& Casteleti, C.H. 2005. Estado da biodiversidade da Mata Atlântica brasileira. In: Galindo-Leal, C. \& Câmara, I.G. (eds.). State of the hotspots - Mata Atlântica: biodiversidade, ameaças e perspectivas. Conservação Internacional, Belo Horizonte. Pp. 43-59.

Souza, F.S.; Salino, A.; Viana, P.L. \& Salimena, F.R.G. 2012. Pteridófitas da Serra Negra, Minas Gerais, Brasil. Acta Botanica Brasilica 26: 378-390.
Stehmann, J.R. \& Sobral, M. 2009. Fanerógamas. In: Drummond, G.M.; Martins, C.S.; Greco, M.B. \& Vieira, F. (eds.). Biota Minas - Diagnóstico do conhecimento sobre a biodiversidade no estado de Minas Gerais, subsídio ao programa Biota Minas. Fundação Biodiversitas, Belo Horizonte. Pp. 355-374.

Tabarelli, M.; Pinto, L.P.; Silva, J.M.C.; Hirota, M.M \& Bedê, L.C. 2005. Desafios e oportunidades para a conservação da biodiversidade na Mata Atlântica brasileira. Megadiversidade 1: 132-138.

Thiers, B. 2011 (continuously updated). Index Herbariorum: A global directory of public herbaria and associated staff. New York Botanical Garden's Virtual Herbarium. Disponível em <http://sweetgum.nybg.org/ih/>. Acesso em 20 Abr 2011.

Uagoda, R.E.S.; Avelar, A.S. \& Coelho Netto, A.L. 2006. Contribuição à geomorfologia cárstica em rochas quartzíticas: médio vale do rio Preto. In: VI Simpósio Nacional de Geomorfologia/Regional Conference on Geomorphology, 2006, Goiânia. Disponível em $<$ http://www.labogef.iesa.ufg.br/links/sinageo/aut/ articles/228.pdf $>$. Acesso em 20 Set 2011.

Valente, A.S.M.; Garcia, P.O.; Salimena, F.R.G. \& OliveiraFilho, A.T. 2011. Composição, estrutura e similaridade florística da Floresta Atlântica, na Serra Negra, Rio Preto - MG. Rodriguésia 62: 321-340. 